\title{
A 1-to-8 Fully Modular Stacked SIW Antenna Array for Millimeter-Wave Applications
}

This paper was downloaded from TechRxiv (https://www.techrxiv.org).

LICENSE

CC BY-NC-SA 4.0

SUBMISSION DATE / POSTED DATE

$11-01-2022$ / 14-01-2022

\section{CITATION}

Segura-Gómez, Cleofás; Palomares-Caballero, Ángel; Padilla, Pablo (2022): A 1-to-8 Fully Modular Stacked SIW Antenna Array for Millimeter-Wave Applications. TechRxiv. Preprint. https://doi.org/10.36227/techrxiv.18134057.v1

$\mathrm{DOI}$

10.36227/techrxiv.18134057.v1 


\title{
A 1-to-8 Fully Modular Stacked SIW Antenna Array for Millimeter-Wave Applications
}

\author{
Cleofás Segura-Gómez, Ángel Palomares-Caballero, and Pablo Padilla
}

\begin{abstract}
This paper presents a vertically stacked SIW antenna array that enables different array configurations with the minimum number of SIW layers. This achievement lies in the modular feature offered by the proposed design. Specifically, 4 distinct array configurations can be produced with only 3 different design of SIW layers. Depending on the number of SIW layers employed in the stacked antenna, the directivity in the E-plane of radiation is modified. To obtain an equal and in-phase power distribution among the array elements, $H$ - and E-plane corporate feeding networks are efficiently implemented in each array configuration. Array configurations of 1, 2, 4 and 8 radiating layers are offered by the proposed modular array, where each radiating layer is formed by 8 H-plane SIW horn antennas. The simulated directivity for the array configurations ranges from $15.8 \mathrm{dBi}$ to $23.8 \mathrm{dBi}$ and the main beam direction remains fixed along the operating frequency band. The array design has been manufactured and proper agreement between simulated and measured results are observed. The measured impedance bandwidth in all the array configurations is from $35 \mathrm{GHz}$ to $41 \mathrm{GHz}(15.79 \%$ bandwidth) with a reduction in the E-plane beamwidth as the number of radiating layers increases.
\end{abstract}

Index Terms-Antenna array, millimeter-wave frequencies, modularity, SIW technology.

\section{INTRODUCTION}

$\mathbf{F}$ OR next generation communication systems, there will be an increase in their operating frequency due to the need to avoid saturated sub-6 GHz bands and to achieve higher bandwidths [1], [2]. However, the increase in frequency is also an engineering challenge because the propagation losses grow substantially [3]. The solutions usually proposed are the reduction of communication links, for example, introducing femtocells [4]; and/or using high directivity antennas to efficiently radiate power [5], [6].

Since the target operating frequencies will be in the millimeter-wave range, the technology used to design the antenna must provide transmission efficiency, be low cost and with a profile as low as possible. One of the technologies that meets mostly these requirements is the substrate integrated waveguide (SIW) [7]. Several antenna arrays have been developed in this waveguide technology for millimeter-wave

This work was supported in part by the Spanish Government under Project PID2020-112545RB-C54, in part by "Junta de Andalucía" under Project B-TIC-402-UGR18, Project A-TIC-608-UGR20, Project P18.RT.4830 and Project PYC20-RE-012-UGR, and in part by the Predoctoral Grants FPU20/00256 and FPU18/01965. (Corresponding author: Cleofás SeguraGómez.)

C. Segura-Gómez, Á. Palomares-Caballero, and P. Padilla are with the Departamento de Teoría de la Señal, Telemática y Comunicaciones, Universidad de Granada, 18071 Granada, Spain (email: cleofas@ugr.es; angelpc@ugr.es; pablopadilla@ugr.es) applications [8]-[10]. One of the most interesting possibility that offers the SIW is the easy layer stacking to produce multilayer antenna arrays. This design strategy was proposed a long time ago for other types of planar technologies such as microstrip [11]. Due to the arrival of SIW technology and the need to operate at millimeter frequencies, this design strategy has been used to make different antenna designs [12]-[16].

Thanks to the feasibility already shown in the literature of making antennas by stacking SIW layers, an advance in this design approach is to provide modularity. The meaning of modular is that depending on the number of plates stacked, the characteristics of the antenna are different, and always using the highest number of common layers. Modular designs have often been used for 2-D phased antenna array design where each antenna or subarray of antennas is fed independently [17]-[19]. Depending the number of modular antennas are used or the type of modular antenna, different directivity values [17], [19] or bandwidth [18] can be achieved. Instead of modularity in a horizontal plane as presented in the previous works, vertical modularity has also been reported in the literature [20], [21] to produce phased array with endfire radiation. However, the possible connection between the different stacked layers becomes more complex. On the other hand, the modularity in passive antenna arrays has been hardly explored. In [22], a vertical stacking of leaky-wave antennas has been presented to enhance the directivity in the E-plane. Nevertheless, the leakage of each antenna has to be adjusted to obtain a uniform feeding along the array which hampers modularity. The antenna design proposed in [23] achieves a certain degree of modularity by stacking in the E-plane several SIW H-plane horn antennas. A series coaxial feeding is employed in this array but this strategy does not provide uniform and in-phase feeding for the array elements and thus, the main beam is tilted and scanned with the frequency.

In this paper, we present a fully modular design of a SIW antenna array stacked in the E-plane. The antenna design provides 4 array configurations with the only use of 3 different types of SIW layers. Depending on the directivity desired for the application or coverage needed, an array configuration is adopted. In addition, due to the fact that the array configurations share layers, this leads to a saving in mass production. To the best of the authors' knowledge, it is the first time that modularity is implemented in an SIW passive antenna array providing different directivity in the E-plane with fixed radiating direction. Fig. 1 shows the design of the proposed modular array. The configuration illustrated in the figure corresponds to an array configuration that provides 


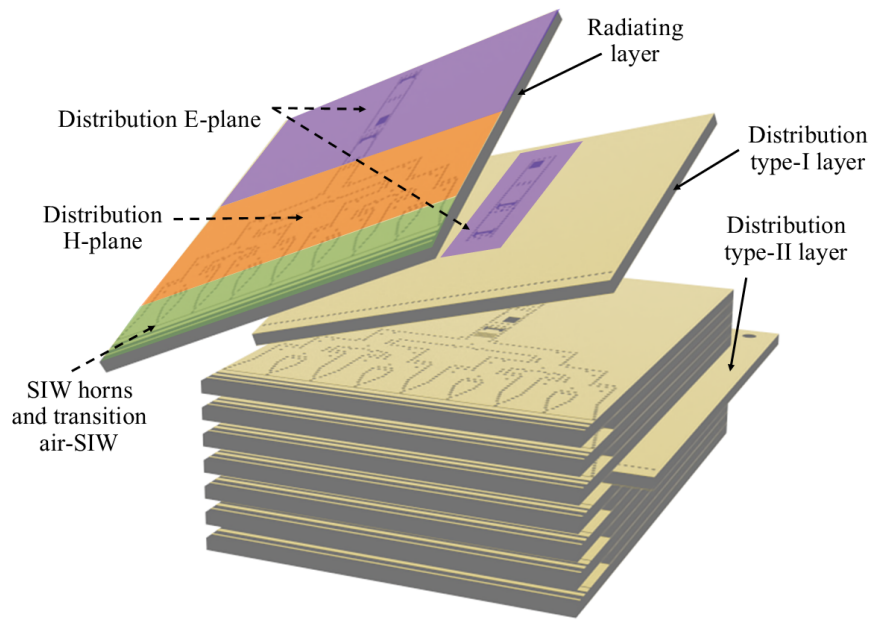

Fig. 1. Design of the proposed fully modular antenna array. The array configuration illustrated is the higher one in terms of stacked antenna elements.

the highest directivity because the antenna array is composed by 8 radiating layers, each one containing 8 SIW horns. Additionally, only two types of distribution layers are needed to obtain equal power distribution from the input port for the four array configurations mentioned above. The document is organized as follows. Section II explains in detail the design and the performance of all the array antenna components. Section III describes the measurement and characterization of the prototypes and also, a comparison and discussion of the measured results are carried out. Finally, Section IV presents the conclusions of the work.

\section{Antenna ARray Design}

The substrate employed for all the designed SIW layers is the commercial RO4003C [24] with double metallized cladding whose thickness is $35 \mu \mathrm{m}$. The height $h_{0}$ of the substrate is $1.524 \mathrm{~mm}$ with a relative permittivity $\varepsilon_{r}$ of 3.55 and tabulated loss tangent $\tan \delta$ of 0.0027 (@10 GHz). By setting a width $w_{0}$ for the SIW waveguide equal to $3.2 \mathrm{~mm}$ and following the general SIW design equations to avoid leakage losses [25] from $32 \mathrm{GHz}$ to $44 \mathrm{GHz}$, the periodicity between metallized vias $d$ and the vias radius $r$ are $0.8 \mathrm{~mm}$ and $0.25 \mathrm{~mm}$, respectively. In the following subsections, each component that composes the antenna array is described and its simulated performance is discussed. All the simulations presented in the paper have been performed with CST Studio Suite.

\section{A. H-plane corporate feeding network and antenna element}

In this first subsection, it is presented the H-plane corporate feeding network that is implemented in the radiating layer (see Fig. 1). This type of corporate feeding network has been used in other works such as [26] but for our design, the bends and power splitters have been designed to cover the target frequency range with a very low reflection coefficient $(-20 \mathrm{~dB})$. In this manner, the possible mismatch will be minimized when all the fundamental components are joined together to form the corporate feeding network. The designed

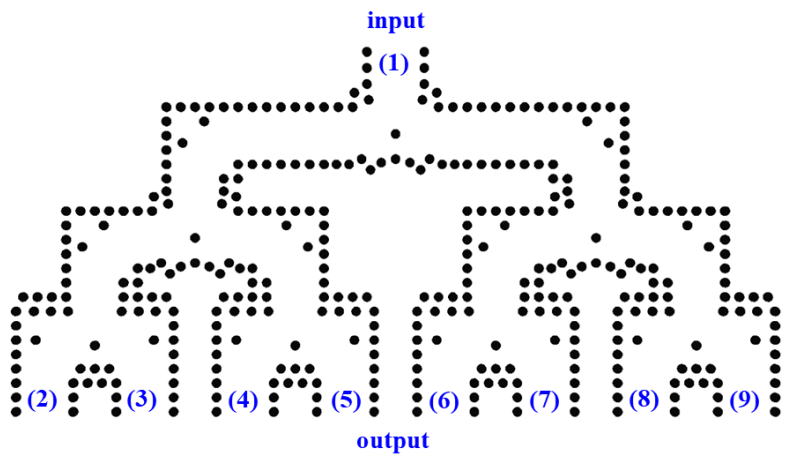

(a)

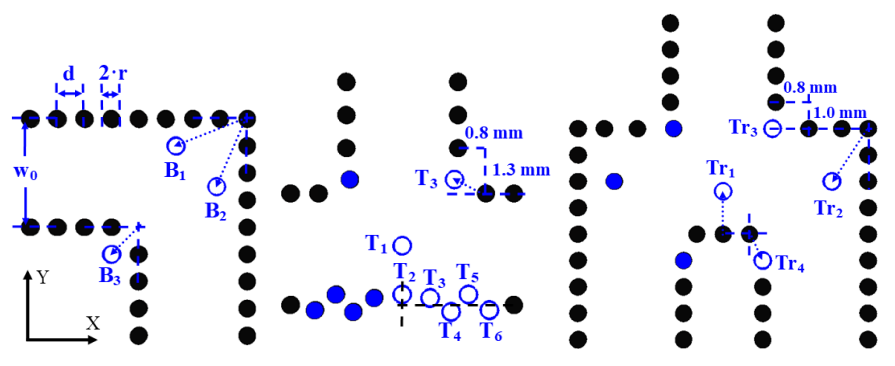

(b)

(c)

(d)

Fig. 2. (a) Layout of the H-plane corporate feeding network and its fundamental components: (b) SIW $90^{\circ}$ bend, (c) SIW $-3 \mathrm{~dB}$ T-splitter, and (d) $-3 \mathrm{~dB}$ compact SIW T-splitter. The blue unfilled vias show the modified or introduced vias and the blue filled vias are the symmetrical vias. The dimensions of the bend are: $\mathrm{B}_{1 \mathrm{X}}=2.1, \mathrm{~B}_{1 \mathrm{Y}}=0.8, \mathrm{~B}_{2 \mathrm{X}}=0.9, \mathrm{~B}_{2 \mathrm{Y}}=2$, and $\mathrm{B}_{3 \mathrm{X}}=\mathrm{B}_{3 \mathrm{Y}}=0.8$. The dimensions of the T-splitter are: $\mathrm{T}_{1 \mathrm{Y}}=1.7, \mathrm{~T}_{2 \mathrm{Y}}=\mathrm{T}_{5 \mathrm{Y}}$ $=0.3, \mathrm{~T}_{3 \mathrm{Y}}=0.2, \mathrm{~T}_{4 \mathrm{Y}}=\mathrm{T}_{6 \mathrm{Y}}=-0.2, \mathrm{~T}_{3 \mathrm{X}}=0.8, \mathrm{~T}_{4 \mathrm{X}}=1.4, \mathrm{~T}_{5 \mathrm{X}}=1.9$, and $\mathrm{T}_{6 \mathrm{X}}=2.5$. The dimensions of the compact T-splitter are: $\operatorname{Tr}_{1 \mathrm{Y}}=1.3, \mathrm{Tr}_{2 \mathrm{X}}=$ 1.1, $\operatorname{Tr}_{2 \mathrm{Y}}=1.6, \operatorname{Tr}_{3 \mathrm{X}}=1.1, \operatorname{Tr}_{4 \mathrm{X}}=0.4$, and $\operatorname{Tr}_{4 \mathrm{Y}}=0.8$. All dimensions are in $\mathrm{mm}$.

feeding network considers one SIW input port and eight SIW output ports which will correspond to the antenna input ports. In order to distribute the electromagnetic (EM) field from the input port to outputs ports, three levels of twoby-two divisions are needed as is illustrated in Fig. 2(a). In the design process, it is important to take into account the distance between the centers of the outputs because they will determine the distance between antenna centers. Therefore, in order to avoid grating lobes and preserve high directivity for the radiating layer, this distance is $5.6 \mathrm{~mm}$. This leads to the preservation of the electrical distance between array elements from $0.6 \lambda_{L}$ to $0.82 \lambda_{H}$ in the considered frequency range. The fundamental components that forms the H-plane corporate feeding network are the $90^{\circ}$ waveguide bend, the $-3 \mathrm{~dB}$ Tsplitter and, the compact design of a T-splitter with waveguide bends. Their layouts are illustrated in in Figs. 2(b), 2(c) and, 2(d), respectively. The T-splitters with waveguide bends are needed in the last splitting level to ensure the distance between centers of the output ports.

In the layout of the fundamental components (Figs. 2(b), 2(c) and, 2(d)), some vias are highlighted in blue since they are very important to achieve a high performance in the scattering parameters (S-parameters). For instance, the SIW bend uses the marked $B_{1}$ and $B_{2}$ vias to keep the effective waveguide width constant during the bend and the offset $B_{3}$ via to avoid the sharp corner. The S-parameters of the depicted fundamen- 


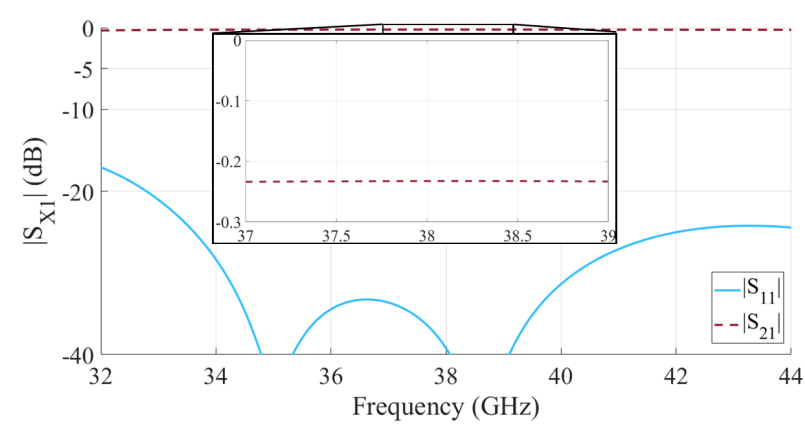

(a)

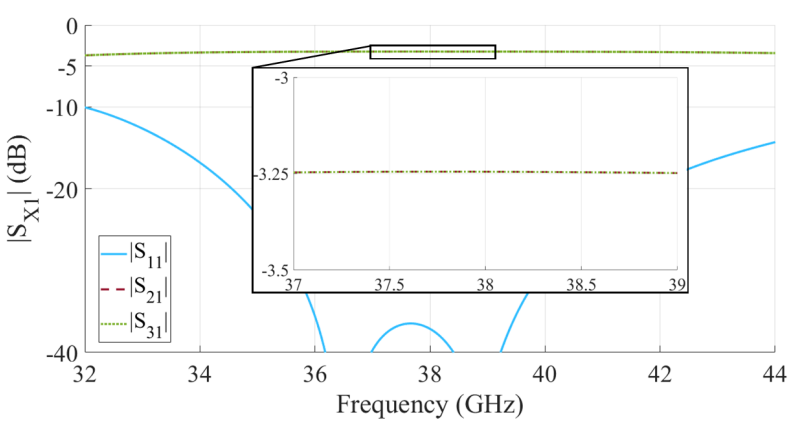

(b)

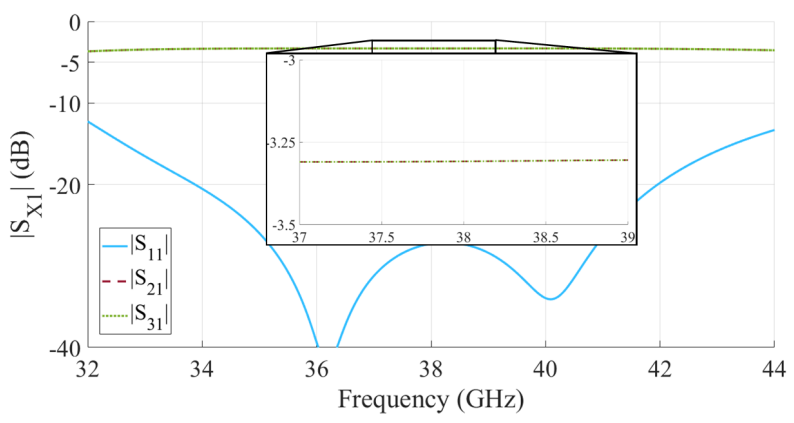

(c)

Fig. 3. $|\mathrm{S}|$-parameters of H-plane components: (a) SIW $90^{\circ}$ bend, (b) SIW $-3 \mathrm{~dB}$ T-splitter, and (c) $-3 \mathrm{~dB}$ compact SIW T-splitter.

tal components are displayed in Fig. 3. All of them show a reflection coefficient $\left|S_{11}\right|$ similar or lower than $-20 \mathrm{~dB}$ from 35 $\mathrm{GHz}$ to $42 \mathrm{GHz}$. Complementary, the transmission coefficient $\left|S_{21}\right|$ is almost the ideal value for all the designs but with some losses due to the presence of the dielectric of the SIW. Once the fundamental elements of the corporate feeding network are designed with the expected performance, all of them are joined to form the final design of the $\mathrm{H}$-plane corporate feeding network shown in Fig. 2(a). The S-parameters in magnitude and phase of the corporate feeding network are presented in Figs. 4(a) and 4(b), respectively. Thanks to the adequate individual performance of the fundamental components, the global performance of the H-plane corporate feeding network maintains a low reflection coefficient in the desired frequency band. This causes a uniform and in-phase feeding through the output ports. As it was observed for the transmission coefficient of the fundamental components, the transmission coefficients of the complete feeding network are a little bit far from the ideal ones due to the dielectric losses.

At the end of each of the eight outputs of the H-plane corpo-

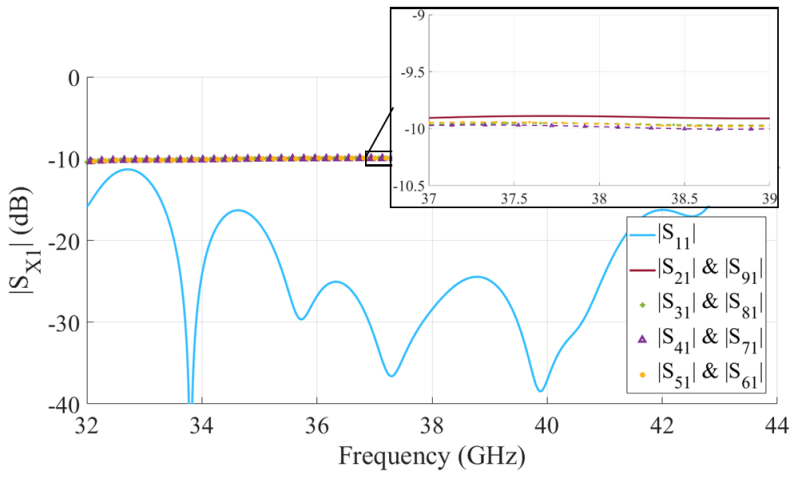

(a)

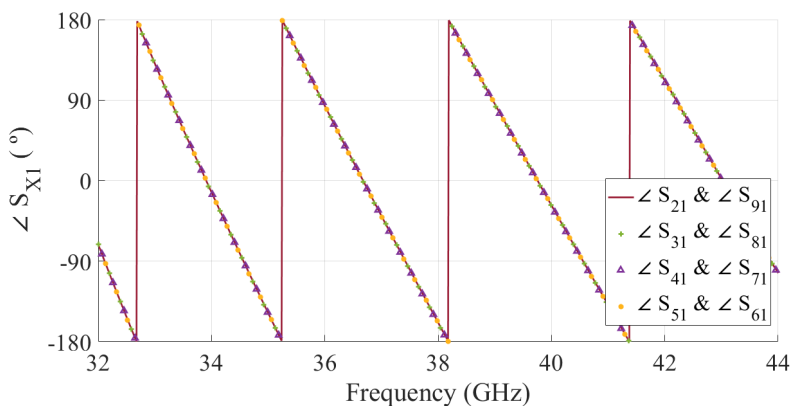

(b)

Fig. 4. $|\mathrm{S}|$-parameters of the H-plane corporate feeding network in (a) magnitude and (b) phase.

rate feeding network, the radiating elements are implemented. These are SIW H-plane horns whose design is shown in Fig. 5(a). They consist of a tapper in SIW waveguide width from $w_{0}$ to the maximum aperture size set by the distance between array elements $(5.6 \mathrm{~mm})$. The angle of flare is fixed by the slant length $L_{H}$. For a proper impedance matching between SIW and free space, a transition is employed. This transition is composed of the dielectric slab and four pairs of metallized - non-metallized strips with sizes $L_{P}$ [23], [27]. In order to use efficiently the geometrical space between SIW horns, the last vias of each SIW horn are shared with the neighboring antennas. In Fig. 5(b), it is presented the reflection coefficient and directivity of the antenna element. A proper impedance matching is achieved on the target frequency band with a directivity of $8 \mathrm{dBi}$ at the center frequency.

\section{B. Modular E-plane corporate feeding network}

This subsection is devoted to describe the waveguide components implemented in the distribution type-I layer (see Fig. 1). This SIW layer enables the uniform and in-phase feeding from the distribution type-II layer to all the stacked radiating layers. Fig. 6 illustrates how the three types of SIW layers are stacked to produce all the array configurations. It is important to note that in the radiating layer it is also implemented waveguide components since in this manner, the number of different layers used to achieve all the array configurations is minimized. The radiating layer is composed of the $\mathrm{H}$ plane distribution (in gray), a waveguide type-III (in green), a waveguide type-I (in blue) and a waveguide type-III from left to right. The distribution type-I layer is composed of two 


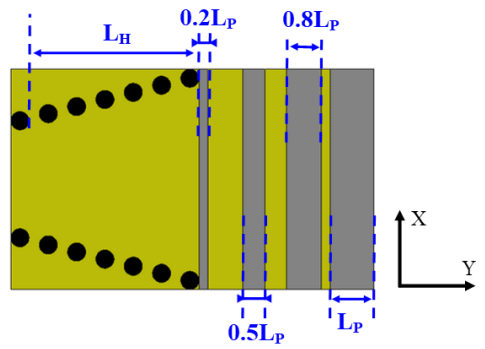

(a)

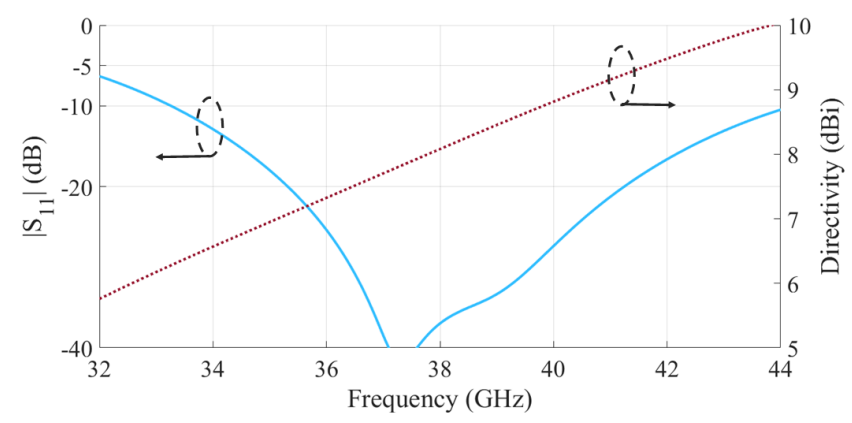

(b)

Fig. 5. SIW H-plane horn: (a) Top view, and (b) $\left|\mathrm{S}_{11}\right|$ and directivity. Dimensions are: $\mathrm{L}_{\mathrm{H}}=4.9 \mathrm{~mm}$, and $\mathrm{L}_{\mathrm{P}}=1.2 \mathrm{~mm}$.

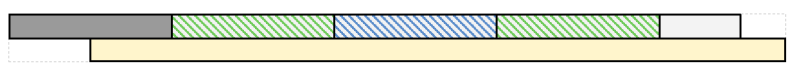

(a)

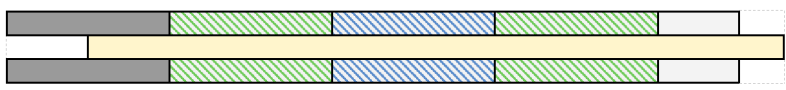

(b)

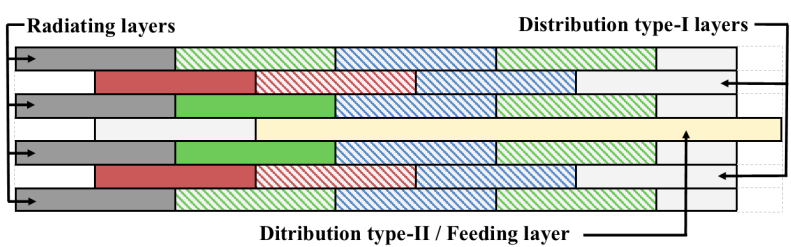

(c)

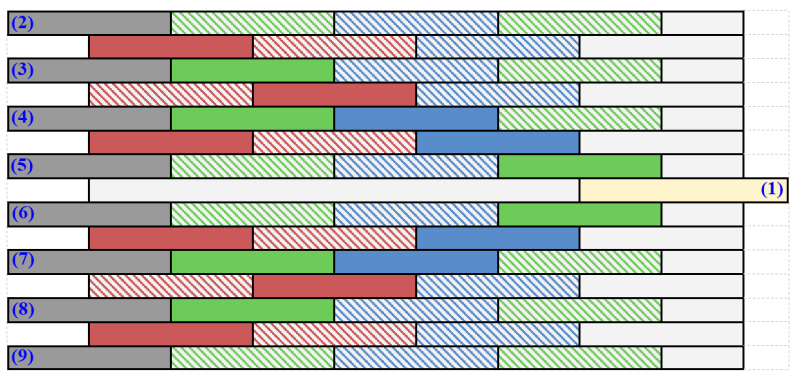

(d)

Fig. 6. Set of array configurations: (a) 1 radiating layer, (b) 2 radiating layers, (c) 4 radiating layers, and (d) 8 radiating layers. The blue color represents the waveguide type-I, the red color represents the waveguide type-II, the green color represents the type-III waveguide, the gray color represents the H-plane distribution and the yellow color represents the feeding waveguide in the distribution type-II layer. The components, which are not striped, are the one used in each array configuration.

waveguides type-II (in red) and a waveguide type-I. The input

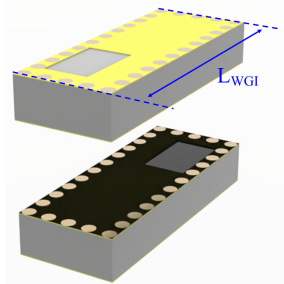

(a)

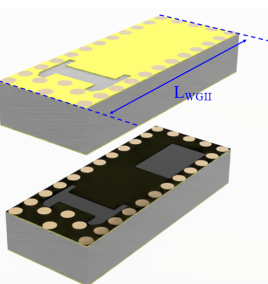

(b)

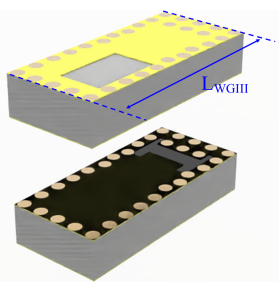

(c)
Fig. 7. Waveguide components used in the modular E-plane corporate feeding network. In each subfigure, it is shown the top view (in yellow) and, bottom view (in black). (a) Waveguide type-I, (b) Waveguide type-II, and (c) Waveguide type-III. The dimensions are: $\mathrm{L}_{\mathrm{WGI}}=9.45 \mathrm{~mm}, \mathrm{~L}_{\mathrm{WGII}}=9.3 \mathrm{~mm}$, and $\mathrm{L}_{\text {WGIII }}=8.35 \mathrm{~mm}$.

port of the antenna array is always located at the distribution type-II layer (in yellow) and at both sides of this layer, the radiating and distribution type-I layers are vertically stacked to produce the desired array configuration. A 3-D view of each waveguide component employed in Fig. 6 is presented in Fig. 7. They are SIW of certain length whose ends present coupling apertures with a particular geometry. These coupling apertures allow the power distribution towards an upper and/or a lower SIW layer of the array. Geometrical details of the designed coupling apertures are shown in Figs. 8(a) and 8(b). The waveguide type-I uses the coupling aperture illustrated in Fig. 8(a) which corresponds to rectangular coupling aperture located near one of the lateral walls of the SIW to enhance EM coupling. The S-parameters of this waveguide component is presented in Fig. 9(a) where it can be observed the satisfactory impedance matching along the desired bandwidth. For the waveguide type-II, whose geometrical details are in Fig. 8(b), a modified version of the conventional dog-bone coupling aperture [28] is employed. This modified dog-bone coupling aperture is etched at both copper layers in order to split the EM field from a middle SIW layer to an upper and lower SIW layers. The S-parameters of this power splitter is presented in Fig. 9(b). It can be observed the fair distribution of the input power to the output ports and the low and wideband impedance bandwidth. The waveguide type-III is composed of the above coupling apertures which have demonstrated an adequate EM performance. Thus, for the sake of conciseness, its S-parameters have been omitted.

In order to use the same radiating layer design for all the array configurations, the array design with a single radiating layer has to receive special attention. As it is illustrated in Fig. 6, the case with a single radiating layer differs in its feeding compared to the other configurations because it is fed by the distribution type-II layer without a power split. For the other array configurations, the radiating layers are always fed by performing a power split in either distribution type-I layer or distribution type-II layer. This fact causes the need of a waveguide component in the radiating layer that enables both feeding depending if only one radiating layer is used or if multiple radiating layers are used. For this purpose, a special waveguide component is designed and it is shown in Fig. 8(c). The waveguide component is composed by the modified dog-bone coupling aperture described above and a 


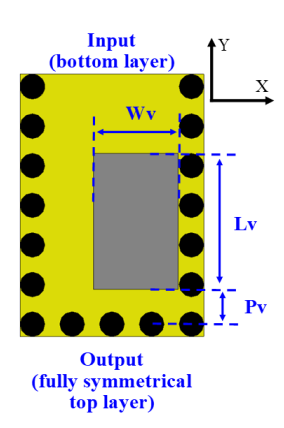

(a)

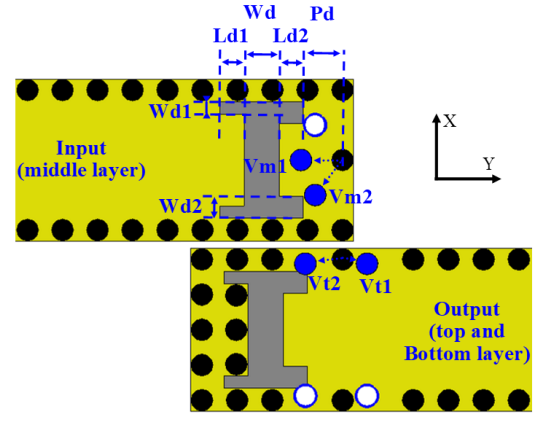

(b)

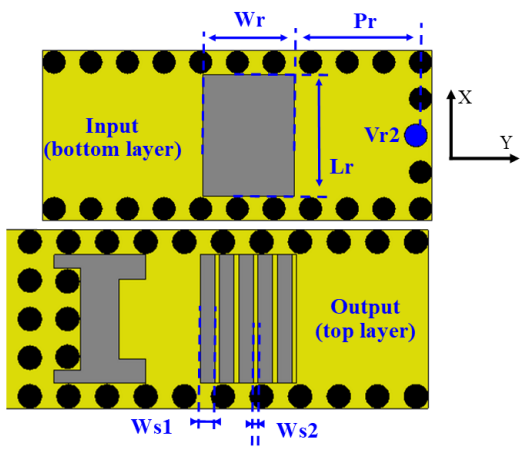

(c)

Fig. 8. Geometric details of coupling apertures used in the waveguide components: (a) rectangular coupling aperture, (b) modified dog-bone coupling aperture, and (c) fragmented rectangular coupling aperture. The dimensions of the rectangular coupling aperture are: $\mathrm{Wv}=1.7, \mathrm{Lv}=2.75$, and $\mathrm{Pv}=0.7$. The dimensions of the modified dog-bone aperture are: $\mathrm{Wd}=0.8, \mathrm{Pd}=1.45$, $\mathrm{Ld} 1=0.55, \mathrm{Ld} 2=0.55, \mathrm{Wd} 1=0.3, \mathrm{Wd} 2=0.5, \mathrm{Vm} 1_{\mathrm{Y}}=0.95, \mathrm{Vm} 2 \mathrm{Y}=$ $0.63, \mathrm{Vt} 1_{\mathrm{X}}=0.1, \mathrm{Vt} 1_{\mathrm{Y}}=0.55, \mathrm{Vt} 2_{\mathrm{X}}=0.1$, and $\mathrm{Vt} 2_{\mathrm{Y}}=0.85$. The dimensions of the fragmented rectangular coupling aperture are: $\mathrm{Wr}=2, \mathrm{Lr}=2.7, \mathrm{Pr}=$ $3.55, \mathrm{Ws} 1=0.3$, Ws2 $=0.1$, and $\mathrm{Vr} 2 \mathrm{Y}=0.1$. All dimensions are in $\mathrm{mm}$.

fragmented rectangular coupling aperture located before the dog-bone aperture. These coupling apertures are implemented in the radiating layer (named top layer in Fig. 8(c)). In the bottom layer and also the feeding layer, a rectangular coupling aperture is implemented allowing the EM coupling between rectangular coupling apertures of both layers. The effect in this EM coupling due to the presence of the dog-bone aperture is minimal since when both layers are stacked, the dog-bone geometry is covered by the copper layer of the bottom layer. This coupling situation described above is the one referred to the case with a single radiating layer where the nonfragmented rectangular coupling aperture is implemented in the distribution type-II layer. The Fig. 9(c) shows the transmission and reflection coefficient of this waveguide component. A suitable operating bandwidth is observed from $35 \mathrm{GHz}$ to $41 \mathrm{GHz}$. For the array configurations that used more than one single radiating layer, the EM coupling to the radiating layer is produced by the dog-bone coupling aperture since the bottom layer is the waveguide type-II. In these cases, the fragmented rectangular coupling aperture has minimal effect because it is covered by copper when the layers are stacked.

Once all the waveguide components that form the modular E-plane corporate feeding network are described, the S-parameters of the most complex array configuration are presented in Fig. 10. It corresponds to the array configuration

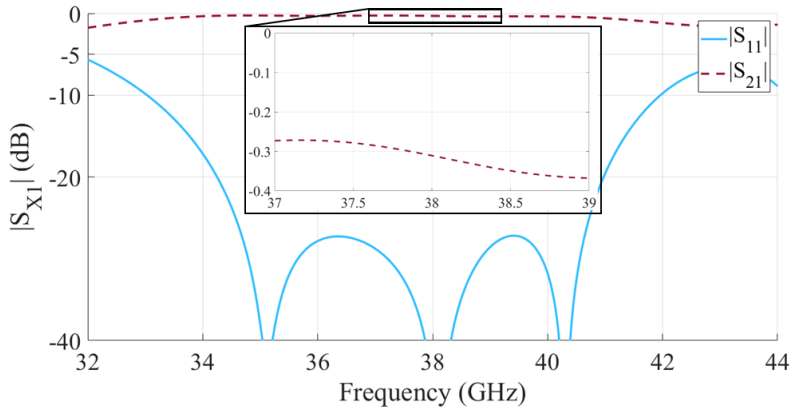

(a)

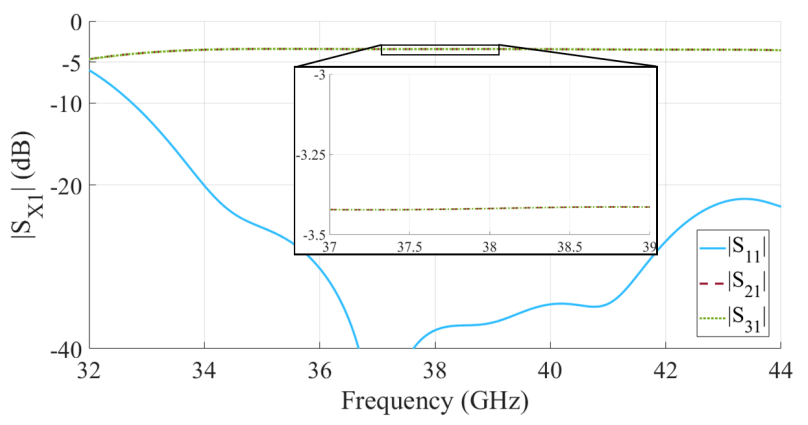

(b)

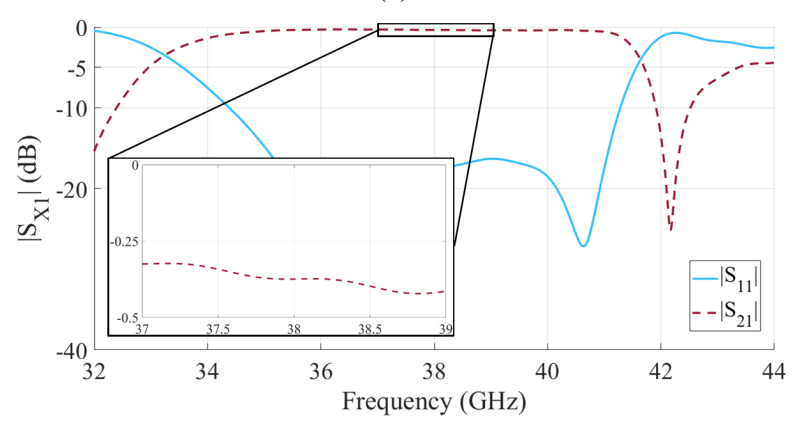

(c)

Fig. 9. $|\mathrm{S}|$-parameters of proposed coupling apertures shown in: (a) Fig. 8(a), (b) Fig. 8(b) and, (c) Fig. 8(c).

when 8 radiating layers are stacked (see Fig. 6(d)). This array configuration involves the largest number of waveguide components presented in this subsection. The S-parameters in magnitude and phase show an impedance matching bandwidth below $-15 \mathrm{~dB}$ from $35 \mathrm{GHz}$ to $42 \mathrm{GHz}$ approximately with a equal and in-phase power distribution among the output ports at the expense of the dielectric losses. Therefore, it is expected that the H-plane SIW horns of the radiating layers provide a high directivity value.

\section{Input port}

This subsection provides a description of the feeding strategy for all the array configurations. The feeding components are implemented in the distribution type-II layer (see Fig. 1). Since the targeted frequency bandwidth is in millimeterwave frequencies and the employed waveguide technology is SIW, a wideband transition from coaxial connector (VNA ports) to SIW is designed. In addition, a commercial endlaunch connector [29] is also utilized between the cable of the VNA and the SIW of the distribution type-II layer to 


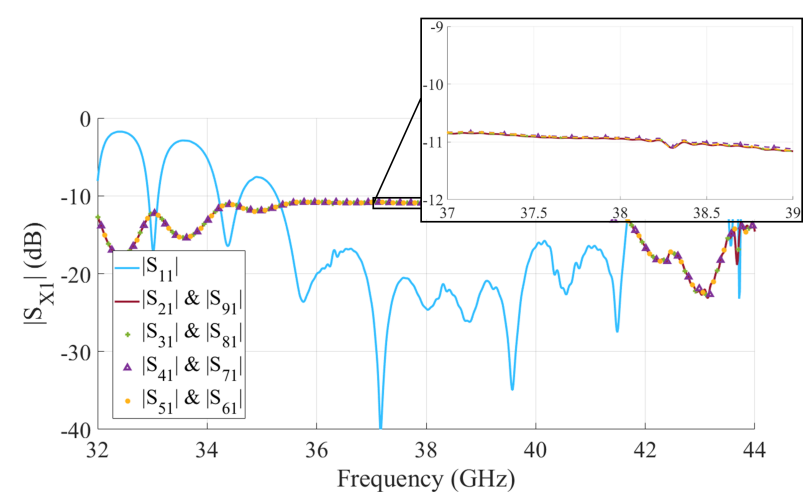

(a)

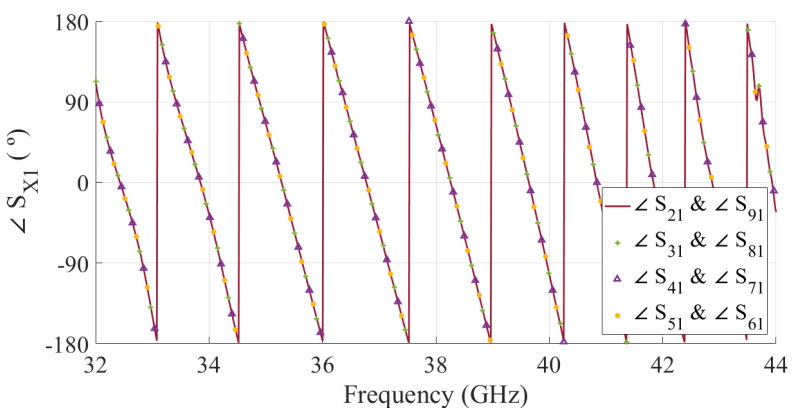

(b)

Fig. 10. $|\mathrm{S}|$-parameters of the array configuration with 8 radiating layers in (a) magnitude and (b) phase.

ease the transition design. Taking into account the design of the end-launch connector, a grounded coplanar-waveguide (GCPW) to SIW transition has been designed. The transition design is based on the work [30]. Nevertheless, a tuning for all the geometrical parameters are required in order to achieve a good transmission and reflection parameters in the desired frequency band and considering the designed SIW and the endlaunch connector geometry. The transition design can be seen in Fig. 11(a) that shows low reflection (under $-20 \mathrm{~dB}$ in the desired frequency band) and high transmission performance as displayed in Fig. 11(b).

A layout of the distribution type-II layer is illustrated in Fig. 11(c) where it can be observed four SIW lines with different lengths whose input port is the above described GCPW-to-SIW transition. At the end of each SIW lines, it is located the modified dog-bone coupling aperture used for the array configuration with 2, 4 and, 8 radiating layers while the rectangular coupling aperture is in one SIW line to the single radiating layer configuration. It is important to remember that for the last case the EM coupling is produced by the fragmented coupling aperture placed at the radiating layer. The reason for the different lengths of the SIW lines of the distribution type-II layer is because the position of the input of the E-plane distribution feeding network is different for each array configuration. In this manner, for all the array configurations, the plane where all the antenna centers are located is preserved as it is clearly observed in Fig. 6. A last geometric detail that can be appreciated in distribution typeII layer (and it is also implemented in the distribution type-I

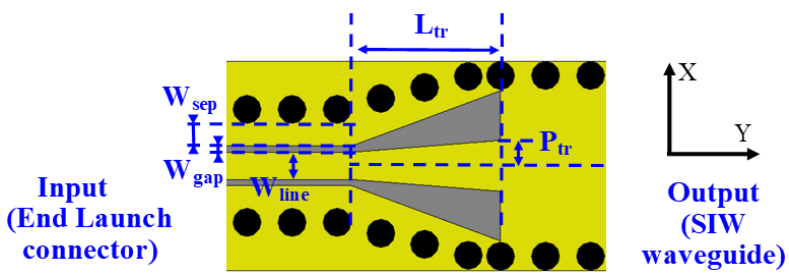

(a)

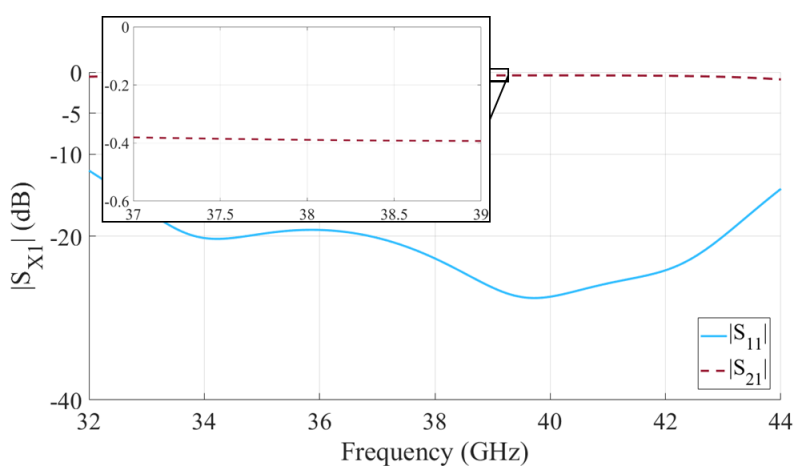

(b)

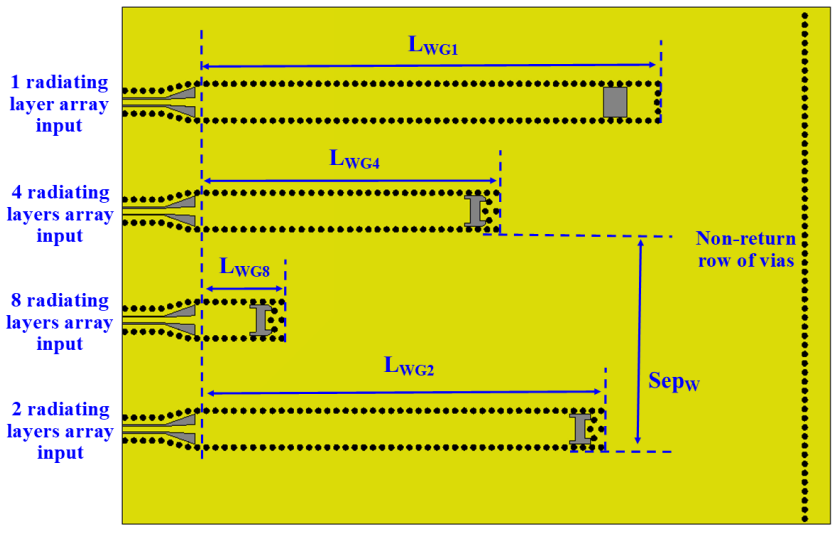

(c)

Fig. 11. Transition from GCPW to SIW: (a) Design, (b) $|\mathrm{S}|$-parameters, and (c) Layout of the distribution type-II layer. The dimensions of GCPW-SIW transition are: $\mathrm{L}_{\mathrm{tr}}=2.4, \mathrm{P}_{\mathrm{tr}}=0.45, \mathrm{~W}_{\text {line }}=0.5, \mathrm{~W}_{\text {sep }}=0.4$, and $\mathrm{W}_{\text {gap }}=0.1$. The dimensions of distribution type-II layer are: $\mathrm{L}_{\mathrm{WG} 1}=40.38, \mathrm{~L}_{\mathrm{WG} 2}=35.4$, $\mathrm{L}_{\mathrm{WG} 4}=26.2, \mathrm{~L}_{\mathrm{WG} 8}=7.4$, and Sep $_{\mathrm{W}}=9.53$. All dimensions are in $\mathrm{mm}$.

layer) is a row of vias near to the right side of the layer. The implementation of these vias improves the directivity for the array configurations because they minimize the back radiation and the effects of coupling between antennas in the different radiating layers.

\section{Simulated performance of array configurations}

Finally, in this subsection, the reflection coefficient and the directivity for all the array configurations are shown in Figs. 12(a) and 12(b), respectively. For these simulated results, the designed components described in subsection II.A are incorporated to the modular E-plane corporate feeding network of each array configuration. The simulated results reveal an impedance bandwidth performance below $-10 \mathrm{~dB}$ for all the array configurations between $35 \mathrm{GHz}$ to $41 \mathrm{GHz}$. It is interesting to point out that for the array configuration different from the single radiating layer case, the impedance bandwidth 


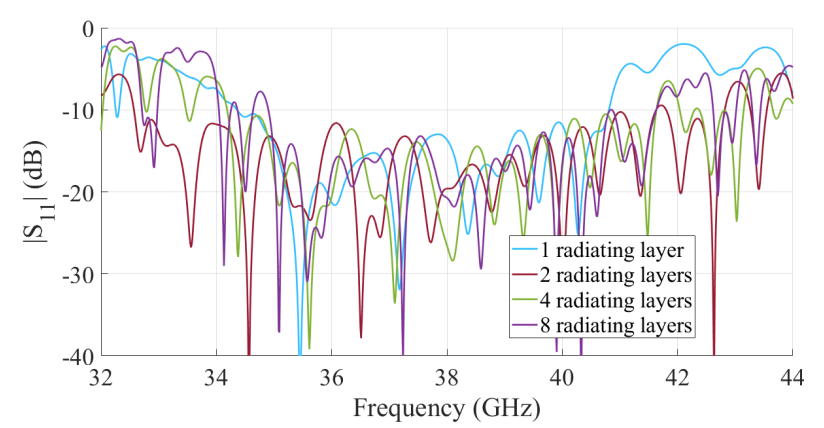

(a)

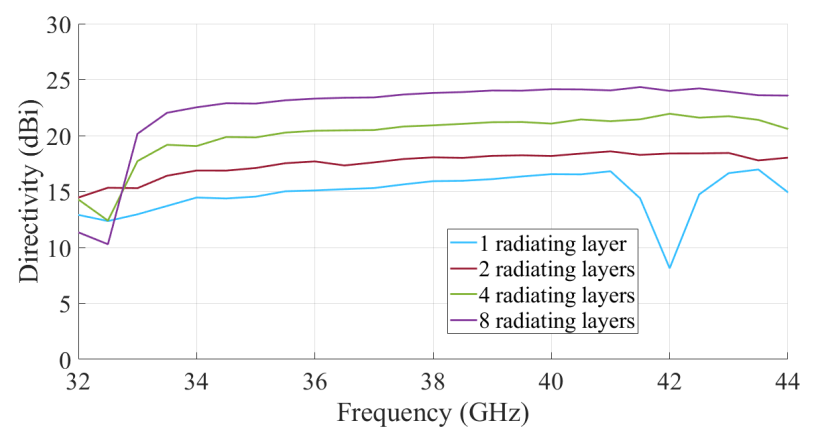

(b)

Fig. 12. Performance of the different array configurations: (a) $\left|S_{11}\right|$, and (b) Directivity.

reaches up to $42 \mathrm{GHz}$. Moreover, in the array configuration with a single radiating layer, a high reflection is observed at $42 \mathrm{GHz}$. This is due to the existence of a strong resonance at this frequency in the waveguide component that uses the fragmented rectangular coupling aperture (see Fig. 9(c)). This fact causes a drop in the directivity at $42 \mathrm{GHz}$ for the case of the single radiating layer, which is observed in the Fig. 12(b). In this figure, it is also shown how the directivity is enhanced when the number of radiating layer increases, producing a linear performance of the directivity in the desired frequency range for all the array configurations.

\section{MeAsurements AND Discussion}

The manufacturing and measurements of the modular SIW antenna array above described are presented in this section. Fig. 13 shows the manufactured SIW layers used in the assembly of all the possible array configurations. As can be seen in the figure, only three different types of SIW layers (radiating, distribution type-I and type-II) are required to manufacture. This fact is a great advantage in a mass production situation because if the same number of multilayer arrays are needed but without modularity, a high number of different layers must be fabricated and it leads to an increase in the price per manufactured SIW layer. The manufactured SIW layers include several alignment and screw holes for a precise vertical stacking in all the array configurations. Besides, the number of screw holes have been optimized so that as many of these are used in each array configuration since for each one, the position of the input port on distribution type-II layer is different.

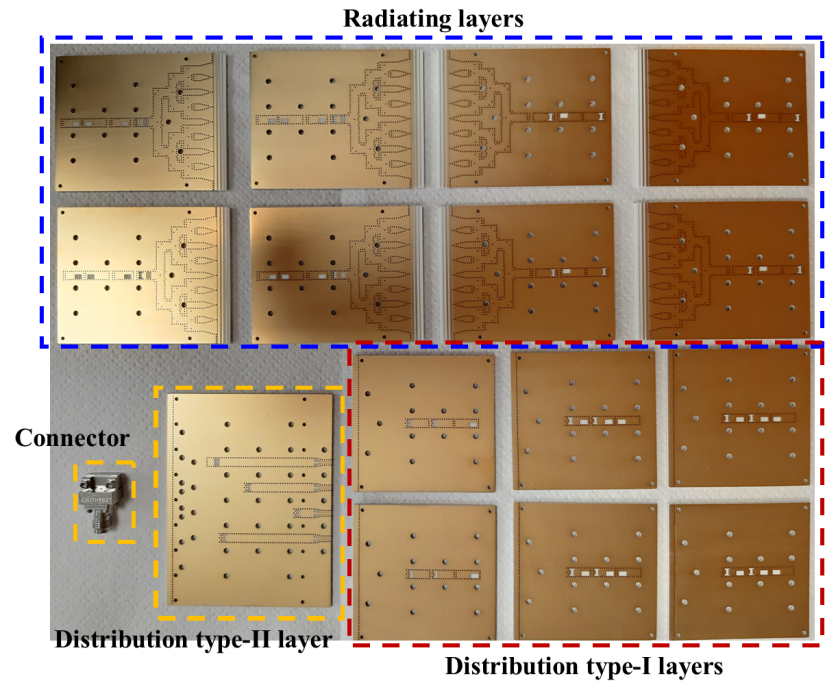

Fig. 13. End-launch connector and manufactured SIW layers that form all the possible array configurations.

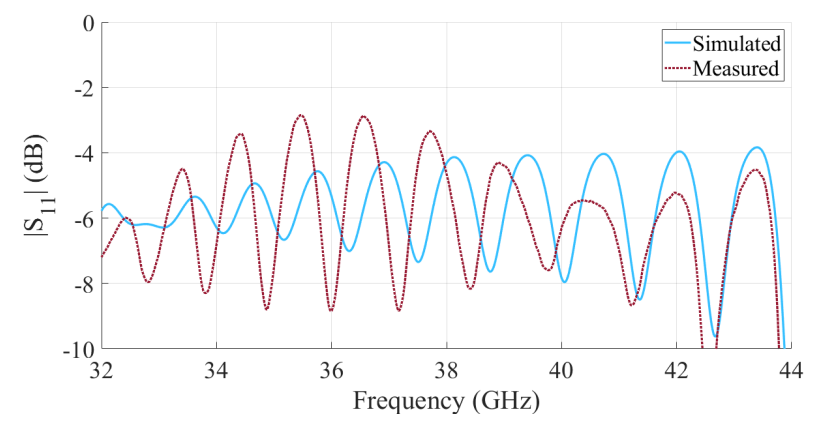

Fig. 14. Simulated and measured $\left|S_{11}\right|$ of short-circuited SIW line set-up.

Before the radiation performance measurement of each array configuration, the dielectric losses are estimated, caused by the dielectric substrate employed in the SIW layers. To calculate the estimated value for the $\tan \delta$ value of the dielectric substrate, a measurement set-up similar to the one presented in [31] to estimate the insertion losses has been adopted. For our case, since we are dealing with SIW technology, the measurement set-up to calculate the insertion losses uses only the longest SIW line of the distribution type-II layer. In the measurement, the rectangular coupling aperture located at the end of the SIW line is short-circuited to produce a total reflection received by the end-launch connector placed at the other end of the SIW line. The expected $\left|S_{11}\right|$ value should be around $0 \mathrm{~dB}$ minus dielectric losses that occur in the SIW line. The simulated and measurement results of this measurement set-up are displayed in Fig. 14. For the simulation, a $\tan \delta$ value of 0.0067 has been set to match both simulated and measured results. This reveals a higher value than expected [24] so that it will produce higher dielectric losses in the designed corporate feeding networks.

The different array configurations are assembled as it is illustrated in Fig. 15. The $\left|S_{11}\right|$ parameter has been measured for all the configurations. The VNA model used in all the measurement process is the R\&S-ZVA67. Fig. 16 presents the simulated and measured results of each array configuration. 


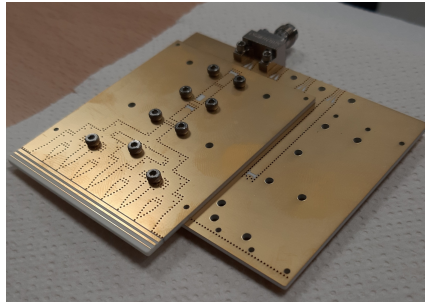

(a)

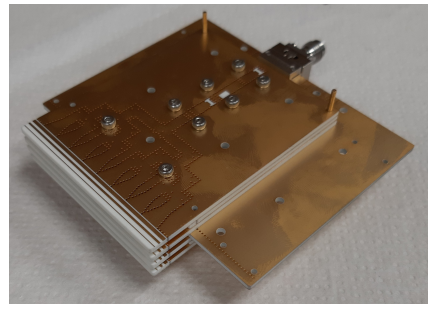

(c)

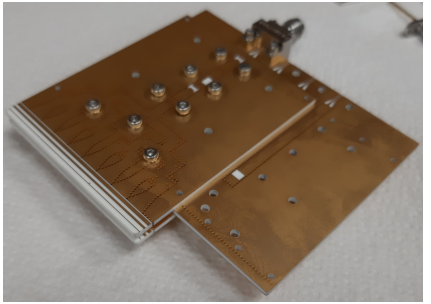

(b)

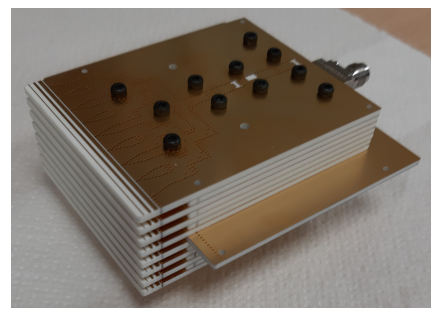

(d)
Fig. 15. Assembled modular arrays. Configuration: (a) 1 radiating layer, (b) 2 radiating layers, (c) 4 radiating layers, and (d) 8 radiating layers.

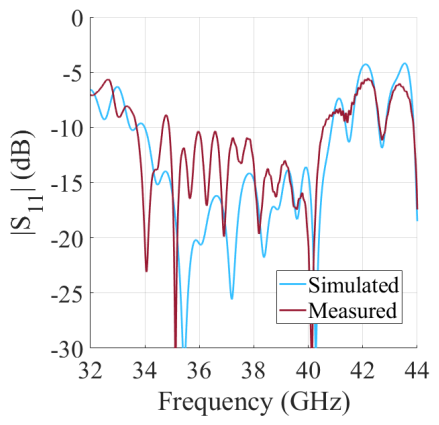

(a)

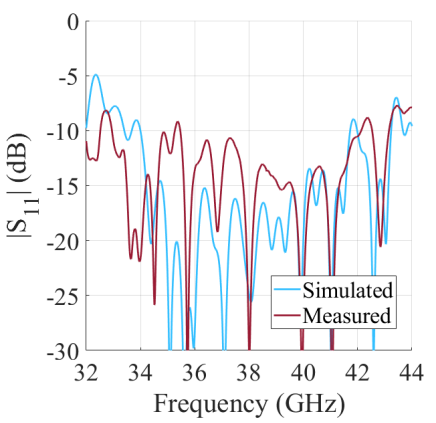

(c)

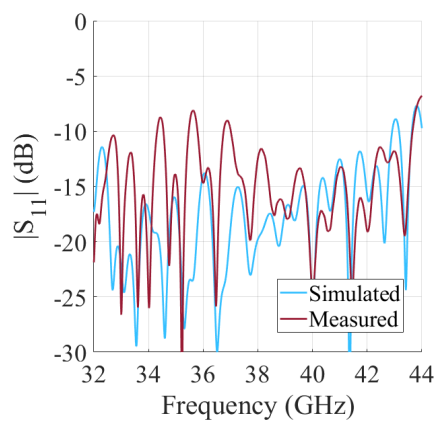

(b)

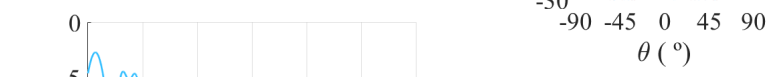

(g)

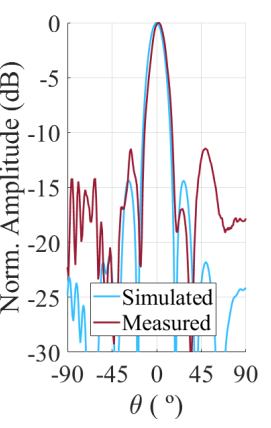

(j)

(d)
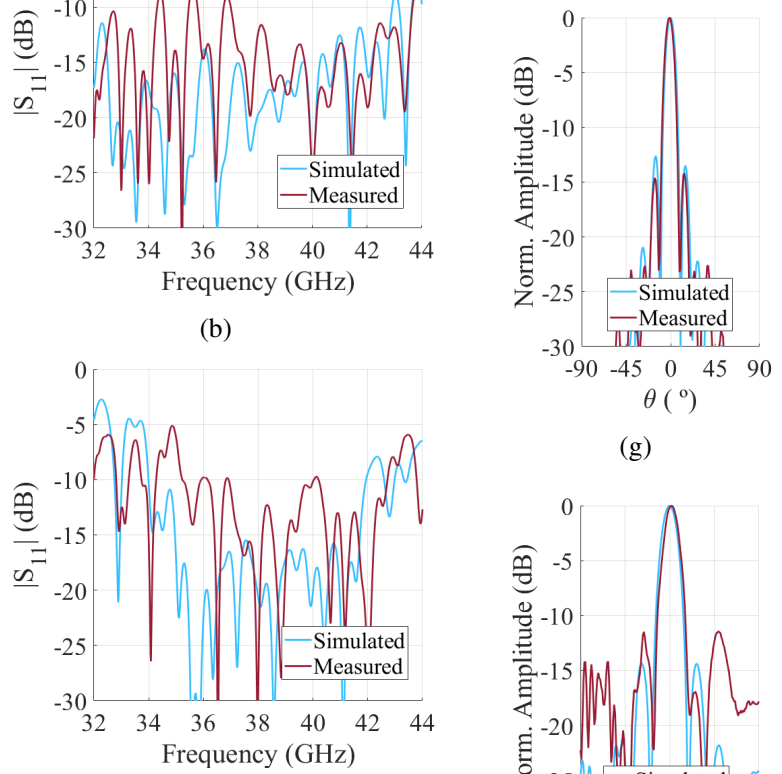

(d)

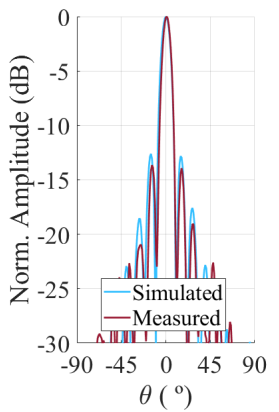

(a)

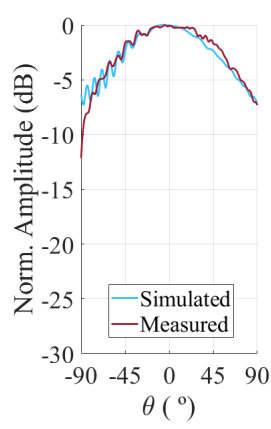

Fig. 16. Simulated and measured $\left|S_{11}\right|$ for the different array configurations: (a) 1 radiating layer, (b) 2 radiating layers, (c) 4 radiating layers, and (d) 8 radiating layers.

The simulated results takes into account the estimated value for the $\tan \delta$ value. As can be seen there is a agreement between the measured and the simulated results. For all the array configurations, the measured impedance bandwidth is the one expected from $35 \mathrm{GHz}$ to $41 \mathrm{GHz}$ (15.79\% bandwidth).

Once the reflection coefficients are measured, the radiation performance of each array configuration is measured in the anechoic chamber of the University of Granada. Fig. 17 presents the simulated and measured results of the H-plane and E-plane radiation patterns at three different frequencies

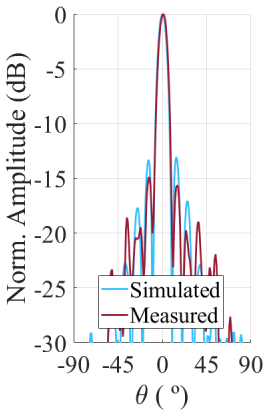

(b)

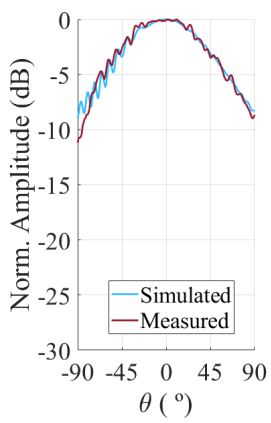

(e)

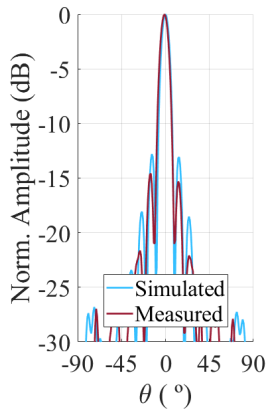

(h)

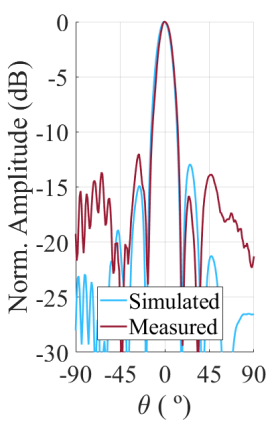

(k)

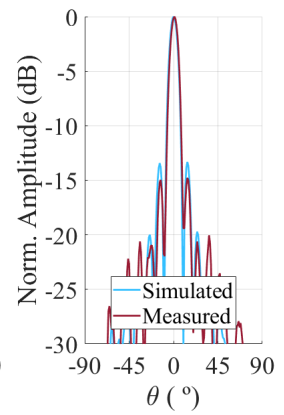

(c)

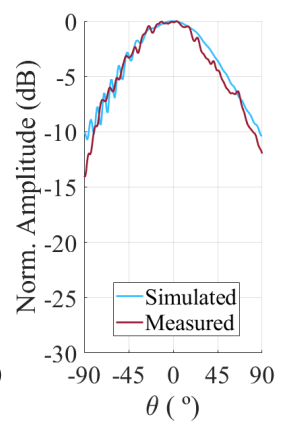

(f)

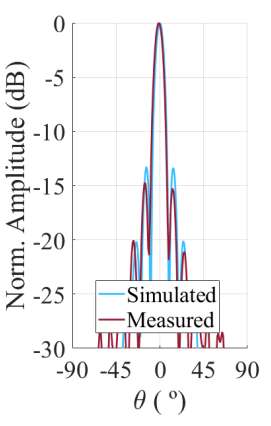

(i)

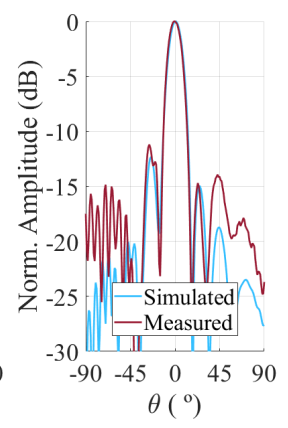

(1)
Fig. 17. Radiation patterns for the 1 radiating layer configuration: (a) $\mathrm{H}$ plane and (d) E-plane at $36 \mathrm{GHz}$. (b) H-plane and (e) E-plane at $38 \mathrm{GHz}$. (c) H-plane and (f) E-plane at $40 \mathrm{GHz}$. Radiation patterns for the 8 radiating layer configuration: (g) H-plane and (j) E-plane at $36 \mathrm{GHz}$. (h) H-plane and (k) E-plane at $38 \mathrm{GHz}$. (i) H-plane and (1) E-plane at $40 \mathrm{GHz}$.

for the 1-radiating layer and 8-radiating layers configurations. Complementarily, in Fig. 18, it is illustrated the 3-D radiation patterns of all the array configurations at the center frequency. Regarding Fig. 17, there is an adequate agreement between the simulations and the measurements with a stable 


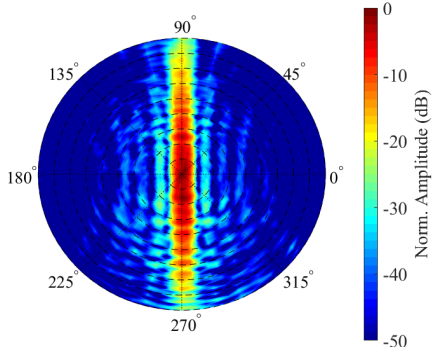

(a)

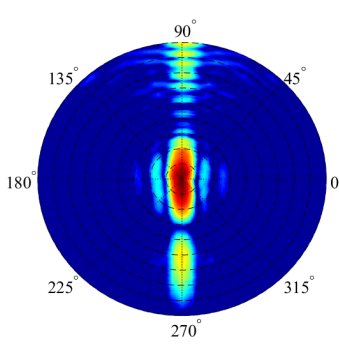

(c)

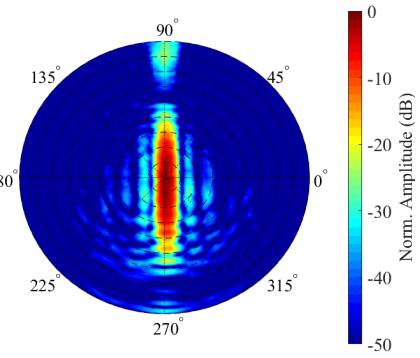

(b)
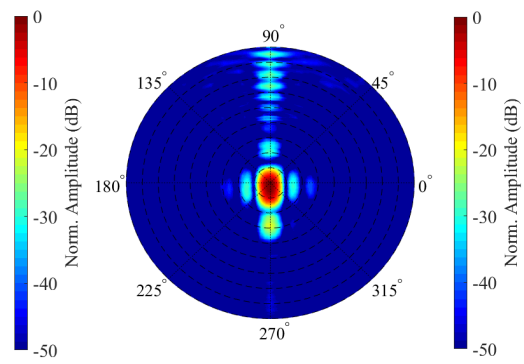

(d)
Fig. 18. Measured 3-D radiation pattern at $38 \mathrm{GHz}$ : (a) 1 radiating layer, (b) 2 radiating layers, (c) 4 radiating layers, and (d) 8 radiating layers.

radiation pattern shape along the frequency range. The $\mathrm{H}$ plane radiation pattern is similar for both array configurations since the number of SIW horns is maintained along the $\mathrm{H}$ plane of radiation. On the contrary, for the E-plane radiation patterns, it is observed a noticeable narrowing between both array configurations. This is due to the increase of antenna elements in the E-plane of radiation when the radiating layers are stacked. The straightforward consequence of this beam narrowing is a high increase in the directivity, as it was observed in Fig. 12(b). The progressive narrowing of the main beam in the E-plane can be seen most clearly in Fig. 18, where the intermediate configurations are shown. For the sake of brevity, only a single frequency is presented since the adequate performance of the radiation patterns along the frequency has been demonstrated in Fig. 17.

Fig. 19 shows the simulated and measured results of the gain for all the array configurations. It can be observed the agreement between the simulated and measured gain values for all the configurations. Moreover, there is an approximately linear increase among the gains as the number of elements is increased by factor of 2 . The gains are stable in the desired frequency band with some linear increase due to the increase of the electrical size of the aperture with frequency. Unfortunately, due to the high value of the $\tan \delta$ value, the gain values are not as high as expected and this fact is magnified as the E-plane corporate network gets larger. Outside the operating frequency band, the gains drop due to impedance mismatching and also due to dielectric losses.

Finally, the Table I discusses the novelty of this work in relation to other SIW antenna arrays at millimeter-wave frequencies. The works included in the comparison table are the most related ones that present a multilayer design with endfire radiation. In [14], a pyramidal horn antenna designed by stacking perforated SIW layers is presented. High

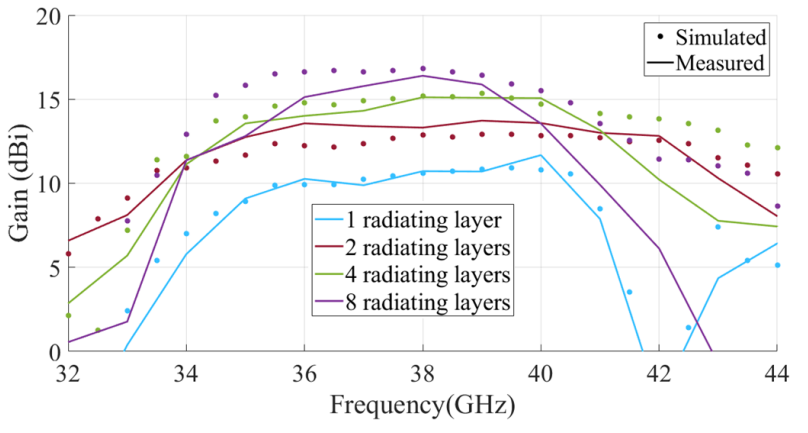

Fig. 19. Simulated and measured gain for all the array configurations.

TABLE I

COMPARISON BETWEEN THE RELATED MULTILAYER SIW ARRAYS

\begin{tabular}{ccccc}
\hline Ref. & $\begin{array}{c}\text { Frequency } \\
\text { band (GHz) }\end{array}$ & $\begin{array}{c}\text { Directivity } \\
(\mathbf{d B i})^{*}\end{array}$ & $\begin{array}{c}\text { Aperture } \\
\text { efficiency }^{\dagger}\end{array}$ & Modularity \\
\hline$[14]$ & 35.1 & 14.6 & $73.84 \%$ & No \\
\hline$[23]$ & $\begin{array}{c}32.9-37 \\
(11.73 \%)\end{array}$ & $11 / 14$ & $48.8 \% / 25.8 \%$ & $\begin{array}{c}\text { Yes } \\
(2 \text { arrays })\end{array}$ \\
\hline This work & $\begin{array}{c}35-41 \\
(15.79 \%)\end{array}$ & $15.8 / 18.1$ & $66.6 \% / 56.6 \%$ & $\begin{array}{c}\text { Yes } \\
\text { (4 arrays) }\end{array}$ \\
\hline
\end{tabular}

* At the center frequency.

$\dagger$ At the center frequency without considering the dielectric losses.

radiation performance with low side lobe levels are achieved but in narrow bandwidth and without modularity. The work in [23] proposes a modular design of vertically stacked $\mathrm{H}$ plane SIW horns. However, the design only offers two array configurations fed by a series coaxial feeding which produces a non-uniform feeding among the antenna elements. Moreover, some beam scanning is observed because of the modifications of the antenna phase feeding along the frequency. The proposed modular SIW antenna array avoids this beam-scanning problem because of the use of corporate feeding networks and also, it provides a great number of array configurations with a wider impedance bandwidth. Therefore, this modular SIW antenna array design provides a low-cost antenna solution due to its modularity to produce a certain directivity in the E-plane for the upper part of the Ka-band.

\section{CONCLusions}

This paper presents a fully modular SIW array design for millimeter-wave frequencies using only 3 different SIW layer components. The total number of array configurations offered by this modular design is 4 with each array configuration producing its own particular directivity. This remarkable fact provides a cost-effective manufacturing in mass production of arrays with different directivities. All the antenna array components have been designed to produce very low-reflection performance in the desired frequency band. This is an important feature to model the $\mathrm{H}$ - and E-plane corporate feeding networks that are implemented in all the array configurations. By the use of modular corporate feeding network in the array design, all the antenna elements are fed uniformly with negligible phase difference among the elements. This keeps 
the main beam direction fixed along the frequency for all the array configurations. Prototypes have been manufactured in order to validate the proposed modular array design. The measured results reveal a satisfactory agreement both in the impedance bandwidth and the radiation performance for all the possible array configurations from $35 \mathrm{GHz}$ to $41 \mathrm{GHz}$. A narrowing in the beamwidth of the E-plane is observed when a greater number of radiating layers are used in the stacked array. Nonetheless, due to the dielectric material used in the SIW, the gain is lower than expected but this can be enhanced using other dielectric material with lower loss tangent such as air, in a air-SIW design.

\section{REFERENCES}

[1] T. S. Rappaport, S. Sun, R. Mayzus, H. Zhao, Y. Azar, K. Wang, G. N. Wong, J. K. Schulz, M. Samimi, and F. Gutierrez, "Millimeter wave mobile communications for $5 \mathrm{G}$ cellular: It will work!" IEEE Access, vol. 1, pp. 335-349, 2013.

[2] L. Wei, R. Q. Hu, Y. Qian, and G. Wu, "Key elements to enable millimeter wave communications for $5 \mathrm{G}$ wireless systems," IEEE Wirel. Commun., vol. 21, no. 6, pp. 136-143, 2014.

[3] J. Zhang, X. Ge, Q. Li, M. Guizani, and Y. Zhang, "5G millimeter-wave antenna array: Design and challenges," IEEE Wirel. Commun., vol. 24, no. 2, pp. 106-112, 2016.

[4] V. Chandrasekhar, J. G. Andrews, and A. Gatherer, "Femtocell networks: a survey," IEEE Commun. Mag., vol. 46, no. 9, pp. 59-67, 2008.

[5] Y. Hu, J. Zhan, Z. H. Jiang, C. Yu, and W. Hong, "An Orthogonal Hybrid Analog-Digital Multibeam Antenna Array for Millimeter-Wave Massive MIMO Systems," IEEE Trans. Antennas Propag., vol. 69, no. 3, pp. 1393-1403, 2021.

[6] R. M. Moreno, J. Ala-Laurinaho, and V. Viikari, "Plastic-Filled DualPolarized Lens Antenna for Beam-Switching in the Ka-band," IEEE Antennas Wirel. Propag. Lett., vol. 18, no. 12, pp. 2458-2462, 2019.

[7] F. Xu and K. Wu, "Guided-wave and leakage characteristics of substrate integrated waveguide," IEEE Trans. Microw. Theory Techn., vol. 53, no. 1 , pp. 66-73, 2005 .

[8] C. Di Paola, K. Zhao, S. Zhang, and G. F. Pedersen, "SIW multibeam antenna array at $30 \mathrm{GHz}$ for 5G mobile devices," IEEE Access, vol. 7, pp. $73157-73164,2019$

[9] Y. Li and K.-M. Luk, "Low-cost high-gain and broadband substrateintegrated-waveguide-fed patch antenna array for $60-\mathrm{GHz}$ band," IEEE Trans. Antennas Propag., vol. 62, no. 11, pp. 5531-5538, 2014.

[10] Q. Wu, J. Hirokawa, J. Yin, C. Yu, H. Wang, and W. Hong, "Millimeterwave multibeam endfire dual-circularly polarized antenna array for $5 \mathrm{G}$ wireless applications," IEEE Trans. Antennas Propag., vol. 66, no. 9, pp. 4930-4935, 2018.

[11] N. Herscovici and D. Pozar, "Analysis and design of multilayer printed antennas: a modular approach," IEEE Trans. Antennas Propag., vol. 41, no. 10, pp. 1371-1378, 1993.

[12] Y. Cai, Y. Zhang, Z. Qian, and J. Liu, "A 16-element corporate-feed multilayer SIW cavity-backed slot antenna array," IET Microw. Antennas Propag., vol. 11, no. 12, pp. 1796-1802, 2017.

[13] W. Li, X. H. Tang, and Y. Yang, "A Ka-band circularly polarized substrate integrated cavity-backed antenna array," IEEE Antennas Wirel. Propag. Lett., vol. 18, no. 9, pp. 1882-1886, 2019.

[14] L. Gong, Y. Fu, K. Y. Chan, J. A. Nanzer, and R. Ramer, "An SIW Horn Antenna Fed by a Coupled Mode Emulating Pyramidal Horn Antennas," IEEE Trans. Antennas Propag., vol. 68, no. 1, pp. 33-42, 2020.

[15] J.-W. Lian, Y.-L. Ban, Q.-L. Yang, B. Fu, Z.-F. Yu, and L.-K. Sun, "Planar Millimeter-Wave 2-D Beam-Scanning Multibeam Array Antenna Fed by Compact Siw Beam-forming Network," IEEE Trans. Antennas Propag., vol. 66, no. 3, pp. 1299-1310, 2018.

[16] J.-W. Lian, Y.-L. Ban, Z. Chen, B. Fu, and C. Xiao, "SIW Folded Cassegrain Lens for Millimeter-Wave Multibeam Application," IEEE Antennas Wirel. Propag. Lett., vol. 17, no. 4, pp. 583-586, 2018.

[17] W. Abdel-Wahab et al., "A modular architecture for wide scan angle phased array antenna for K/Ka mobile SATCOM," in 2019 IEEE MTTS International Microwave Symposium (IMS). IEEE, 2019, pp. 10761079.

[18] R. W. Kindt, "Prototype Design of a Modular Ultrawideband Wavelength-Scaled Array of Flared Notches," IEEE Trans. Antennas Propag., vol. 60, no. 3, pp. 1320-1328, 2012.
[19] J. T. Logan, R. W. Kindt, M. Y. Lee, and M. N. Vouvakis, "A New Class of Planar Ultrawideband Modular Antenna Arrays With Improved Bandwidth," IEEE Trans. Antennas Propag., vol. 66, no. 2, pp. 692-701, 2018.

[20] C.-N. Chen et al., "38-GHz Phased Array Transmitter and Receiver Based on Scalable Phased Array Modules With Endfire Antenna Arrays for 5G MMW Data Links," IEEE Trans. Microw. Theory Techn., vol. 69, no. 1, pp. 980-999, 2021.

[21] R. Klimovich, S. Jameson, and E. Socher, "W-Band Endfire 2-D PhasedArray Transmitter Based on $\times 9$ CMOS Active Multiplier Chips," IEEE Trans. Antennas Propag., vol. 68, no. 12, pp. 7893-7904, 2020.

[22] N. Memeletzoglou and E. Rajo-Iglesias, "Array of stacked leaky-wave antennas in groove gap waveguide technology," Sci. Rep., vol. 11, 01 2021.

[23] C. Segura-Gómez, Á. Palomares-Caballero, A. Alex-Amor, J. Valenzuela-Valdés, and P. Padilla, "Modular Design for a Stacked SIW Antenna Array at Ka-Band," IEEE Access, vol. 8, pp. 158 568-158 578, 2020.

[24] RO4000 Series, ROGERS Corporation. [Online]. Available: https://rogerscorp.com/-/media/project/rogerscorp/documents/advancedconnectivity-solutions/english/data-sheets/ro4000-laminates-ro4003cand-ro4350b - data-sheet.pdf

[25] D. Deslandes and $\mathrm{K}$. Wu, "Design consideration and performance analysis of substrate integrated waveguide components," in 2002 32nd European microwave conference. IEEE, 2002, pp. 1-4.

[26] H. Wang, D.-G. Fang, B. Zhang, and W.-Q. Che, "Dielectric Loaded Substrate Integrated Waveguide (SIW) $H$-Plane Horn Antennas," IEEE Trans. Antennas Propag., vol. 58, no. 3, pp. 640-647, 2010.

[27] L. Wang, M. Garcia-Vigueras, M. Alvarez-Folgueiras, and J. R. Mosig, "Wideband H-plane dielectric horn antenna," IET Microw. Antennas Propag., vol. 11, no. 12, pp. 1695-1701, 2017.

[28] S. B. Cohn, "Microwave Coupling by Large Apertures," Proceedings of the IRE, vol. 40, no. 6, pp. 696-699, 1952.

[29] $1.85 \mathrm{~mm}$ Connector-91R60918 Datasheet, Southwest Microwave, Inc. [Online]. Available: https://mpd.southwestmicrowave.com/wpcontent/uploads/2018/06/1892-03A-6.pdf

[30] R. Kazemi, A. E. Fathy, S. Yang, and R. A. Sadeghzadeh, "Development of an ultra wide band GCPW to SIW transition," in 2012 IEEE Radio and Wireless Symposium, 2012, pp. 171-174.

[31] Á. Palomares-Caballero, A. Alex-Amor, J. Valenzuela-Valdés, and P. Padilla, "Millimeter-Wave 3-D-Printed Antenna Array Based on GapWaveguide Technology and Split E-Plane Waveguide," IEEE Trans. Antennas Propag., vol. 69, no. 1, pp. 164-172, 2021. 